\title{
Presentism and black holes
}

\author{
Geurt Sengers ${ }^{1}$
}

Received: 24 April 2015 / Accepted: 18 December 2015 / Published online: 23 April 2016

(C) The Author(s) 2016. This article is published with open access at Springerlink.com

\begin{abstract}
In a recent publication in the European Journal for Philosophy of Science (Romero and Pérez, European Journal for Philosophy of Science, 4, 293-308, 2014), Romero and Pérez claim to reveal new trouble for the already difficult life of presentism in relativistic spacetimes. Their argument purports to demonstrate the impossibility of postulating a viable present in the presence of black holes, in particular the Schwarzschild geometries. I argue that their argument is flawed, and that the Schwarzschild geometries they consider offer no novel threats to presentism. However, if we consider more general black holes, different and more potent threats may be lurking in the dark. I discuss these threats and sketch how a presentist may respond, thus painting a more balanced picture of the way the existence of black holes might impact presentist metaphysics. At present there is no decisive verdict from black holes, but it turns out a close scrutiny of the developments in the relevant physics will be needed to see whether their import will remain so inconclusive.
\end{abstract}

Keywords Presentism · General relativity · Black holes · Philosophy of time

\section{Introduction}

Presentism may still be the metaphysics presumed by common-sense. In the philosophical literature however, it leads a troubled life. The thesis can be understood as the claim that only events in the present exist, while those in future and past do not.

Geurt Sengers

geurtsengers@gmail.com

1 Faculty of Philosophy, Erasmus University Rotterdam, Burg. Oudlaan 50, Room H5-09, 3062

PA Rotterdam, The Netherlands 
The formulation is subject to many subtleties that I will not go into here (see Crisp 2003 or Bourne 2006 for proposals). I will focus here only on a requirement that the more robust and traditional of such formulations share: that of a global, space-like foliation of our spacetime, with each slice representing the world at a moment in time.

To many minds, special relativity alone decisively refutes presentism already. Unattractive as it may be, however, it remains possible to consistently add a present to special relativity (see for instance Rakic 1997). So for the purposes of this paper, I will ask to suspend disbelief and focus only on some further problems that general relativity brings.

That not all spacetimes of general relativity can harbour such a present has been known at least since Gödel (1949) offered a solution of Einstein's field equations that is not everywhere so foliable. But, since it was clear that these solutions do not describe the actual universe, the troubles were at least a modal step removed (Earman 1995; Dorato 2002), and the presentist might still find a sense of safety in that distance.

However, there are other problematic solutions in general relativity that promise to bring similar trouble closer to actuality. Candidate troublemakers are the solutions thought to describe black holes-by now uncontroversially part of the viable models of our actual universe. A recent paper by Romero and Pérez, 'Presentism meets Black Holes', argues that there can be no viable presentism in such geometries. Romero and Pérez should be praised for putting black holes on the agenda in the presentism debate, but I will argue that their argument is flawed.

In brief, Romero and Pérez argue that at the horizon of a black hole, the only viable choice for a present is its (light-like) horizon itself. Since different events on this horizon are co-present with temporally separated distant events, we run into a problem: we must now say that temporally separated events are each co-present with events on the horizon which are mutually co-present. Then, by transitivity of co-presentness, the temporally separated events must also be co-present. This is incompatible with presentism.

First I will show how their argument fails. However, this does not mean presentism is safe. I will dive deeper into black holes, encounter further trouble, and discuss how a presentist might respond. In the exposition that follows, I will limit my focus to the novel problems presentism encounters in black hole geometries. This means that, even if I do sometimes touch upon them, I will not pursue in any depth many problematic issues for presentism in these geometries in so far as they are already present in relativistic context more generally, since then they are not specific to the black hole geometries considered here.

\section{Trouble on the horizon?}

\subsection{A simple black hole}

In general relativity, motion under gravity alone is described as geodesic trajectories in spacetime geometries that are dynamically determined by their matter and energy content. These geometries are described by metrics that are solutions of the 
Einstein field equations. Since light traces null geodesics, the metric determines the behaviour of the lightcones familiar from special relativity: at each event there is a future cone that encloses all events that could be influenced from that event, respecting the speed of light as an upper bound on the propagation of these influences; similarly, there is a past lightcone comprising all events that could have influenced the event. It is between these two cones that, locally, a three-dimensional spacelike slice might be sought that could represent a present. The metric determines how these cones fit together in a larger spacetime. As such their behaviour is determined by matter content-giving rise to gravity as we know it. In the presence of a black hole, the lightcones tilt toward its centre. At the black hole's horizon, the tilt becomes such that any outgoing light-ray aligns with this horizon. Since the trajectories of both light and massive objects are confined to this cone, neither light nor massive objects can move fast enough to escape this horizon, as this would require a speed greater than that of light. Such is it that a black hole begets its colour, or lack thereof. ${ }^{1}$

\subsection{Romero and Pérez's arguments}

Romero and Pérez offer arguments that in the presence of a Schwarzschild black hole, the only viable option for an observer crossing the horizon is to choose the present on the Schwarzschild horizon itself. One can see how this would lead to trouble for presentism. According to the in-falling presentist, the whole of the horizon should exist. Distant presentists will not agree. They will postulate a present that slices the horizon in a particular way. They might, for instance, consider themselves at some time co-present with the event of the first observer passing the horizon. But then, they will maintain part of the horizon still lies in the nonexistent future. So we've run into a contradiction, and presentism takes the fall.

But why do Romero and Pérez claim that the present is to be chosen on the horizon? Their claim is initially suggested by inspecting the behaviour of the lightcones at the Schwarzschild horizon in Schwarzschild coordinates. In these coordinates (setting $c=G=1$ ), the metric reads

$$
d s^{2}=-\left(1-\frac{2 M}{r}\right) d t^{2}+\left(1-\frac{2 M}{r}\right)^{-1} d r^{2}+r^{2} d \Omega^{2} .
$$

We can calculate the behaviour of the lightcones relatively easily by imposing the null condition, $(d s=0)$, obeyed by radial, $(d \Omega=0)$, light-rays. We obtain the following condition.

$$
0=d s^{2}=-\left(1-\frac{2 M}{r}\right) d t^{2}+\left(1-\frac{2 M}{r}\right)^{-1} d r^{2}
$$

\footnotetext{
${ }^{1}$ A somewhat less simplistic sketch would attribute this to the redshift effect.
} 


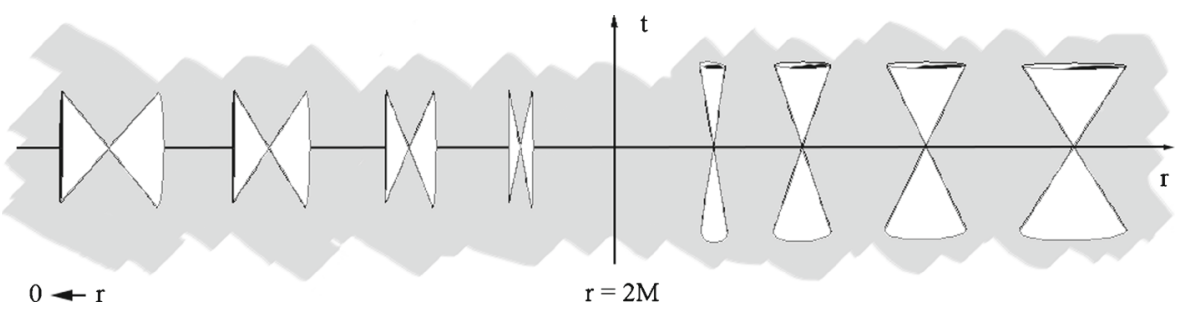

Fig. 1 Behaviour of the lightcones approaching the horizon in Schwarzschild coordinates

From Eq. 2.2 we can easily derive an expression for the behaviour of the slopes $d t / d r$, in these coordinates, of the lightcones as we approach, and go beyond, the horizon:

$$
\frac{d t}{d r}= \pm\left(1-\frac{2 M}{r}\right)
$$

This leads to the familiar diagram in Fig. 1.

In this diagram, we see that the lightcones from the outside in thin out (right), and from the inside out flatten out (left), both leading to a seeming alignment of both the in and outgoing light-rays with the horizon. Now Romero and Pérez take this to imply that any present in between these lightcones must be squashed onto the horizon, as indicated in their Fig. 2.

The figure is suggestive, but it is not all Romero and Pérez offer in support of their claim. Let me quote their arguments:

There is not much to do with [the claim that all events on the Schwarzschild horizon must be simultaneous] since it follows from the condition that defines the null surface: $d s=0 ;{ }^{2}$ [...]. A presentist might refuse to identify 'the present' with a null surface. After all, in Minkowskian space-time or even in a globally time-orientable pseudo-Riemannian space-time the present is usually taken as the hyperplane perpendicular to the local time [...]. But in space-times with black holes, the horizon is not only a null surface, it is also a surface locally normal to the time direction. This can be appreciated in Fig. 2, where the angle $\theta$ is the angle between the null surface and the hyperplane of the present. In Minkowskian space-time such an angle is $45 \mathrm{deg}$ when the speed of light is measured in natural units $(c=1)$. In such a space-time, certainly the plane of the present is not coincident with a null surface. However, close to the event horizon of a black hole, things change, as indicated by Eq. 2.3. As we approach the horizon, $\theta$ goes to zero, i.e. the null surface matches the plane of the present. On the horizon, both surfaces are exactly coincident: $\theta \rightarrow 0$ when $r \rightarrow r_{S c h w}$. A presentist rejecting the identification of the present with a closed null surface on an event horizon should abandon what is perhaps her most cherished belief: the identification of 'the present' with hypersurfaces that are normal to a local time direction.

\footnotetext{
${ }^{2}$ The authors add a footnote: " $d s=c d \tau=0 \rightarrow d \tau=0$, where $d \tau$ is the proper temporal separation." The authors do not specify the proper time separation between what events; but the separation is only zero for events both on the lightcone.
} 


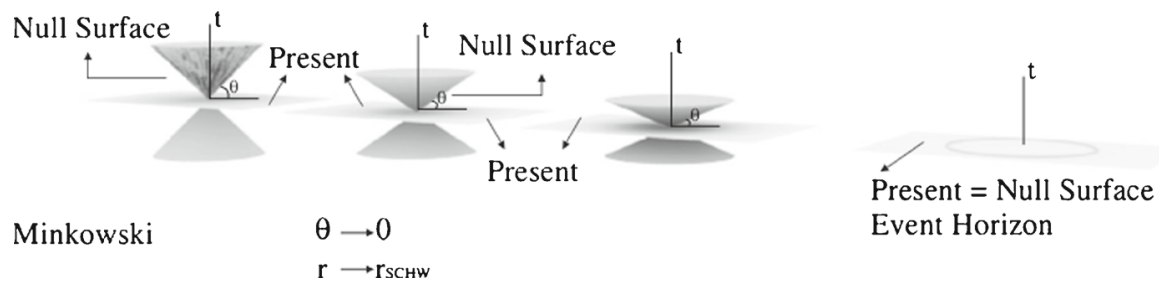

Fig. 2 Figure provided in Romero and Pérez (2014) p.304. Original caption: "Light cones aperture angles at different distances from the horizon of a Schwarzschild black hole. On the horizon the null surface is coincident with the hyperplane of present."

So we can discern two distinct arguments. First, the claim that the lightcones de facto open up onto the horizon upon approach, taking the present with them. Second, the claim that the choice of the present on the horizon is enforced by the requirement of the present's orthogonality to the time direction.

Consider the first argument. First observe that much is made of the behaviour of lightcones in, specifically, Schwarzschild coordinates and their behaviour at the horizon. Let us remember that expression (2.1) for the Schwarzschild metric looks different in different coordinates. This version of the metric becomes degenerate at the horizon $r=2 M$, as $\lim _{r \rightarrow 2 M} g_{r r}$ is infinite, where $g_{r r}=(1-2 M / r)^{-1}$ in Eq. 2.1. The degeneracy, however, is long known to be a mere coordinate artefact. Sometimes it is referred to as a coordinate-singularity, distinguished from real singularities by the fact that they are removable by transforming to different coordinates. The nature of real singularities in general relativity is still under much discussion, but the nature of the Schwarzschild singularity is not. An in-falling observer would locally note nothing strange as he passes the horizon-except ever increasing tidal forces. When using Schwarzschild coordinates, care must be taken not to draw ontological conclusions about space-time that derive from the singular behaviour of coordinates at the horizon.

Now, what is immediately suspicious about the argument is the basic fact of general relativity that we can always move to local inertial frames in which the metric takes the standard Minkowskian form familiar from special relativity. But in these coordinates there remains, of course, plenty of wriggling room in between the lightcones. It is true that for an infalling presentist, the outgoing lightray would lie along the horizon (this is what makes it a horizon; nothing may move faster than this ray). But there is also an infalling lightray that does not, and there is simply a region of spacetime in between them. An in-falling presentist would then have no trouble finding a local spatial present at the horizon. In fact, this is exhibited by any coordinate system that remains regular at the horizon; in the oft-used Eddington-Finkelstein coordinates for instance, the behaviour would look like Fig. 3.

We must take good care to avoid drawing conclusions that depend upon Schwarzschild coordinates, and extrapolation of their use into a region where they are known to become degenerate, namely the horizon. For instance, while it is true to say that $d t / d r$ approaches $\pm \infty$ as $r$ approaches the horizon, it does not makes sense 


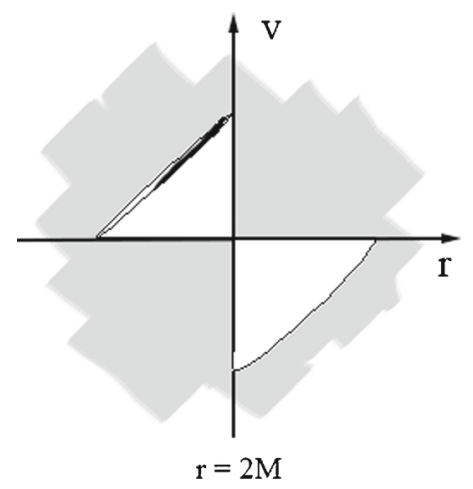

Fig. 3 Behaviour of the lightcones approaching at the horizon in Eddington-Finkelstein coordinates

to use expression (2.2) at the horizon itself, and claim that both ingoing and outgoing light-rays, and hence the lightcones, align with it. The fact that the Schwarzschild- $t$ coordinate tends to infinity for any object passing the horizon, including that of ingoing and outgoing light-rays, makes it hard to see that in fact ingoing and outgoing rays remain distinct there.

The above considerations show that the angle between the lightcones simply is not a coordinate invariant-the expression for the slope is manifestly coordinate dependent. Romero and Peréz do argue correctly that the spatiotemporal character (a distance's or surface's being time-, space-, or light-like) is a coordinate invariant feature. But the invariant light-likeness of the horizon does not suffice for the invariance of the angle. So nor does it suffice for the alignment of both (past and future) lightcones with the horizon. In Minkowski spacetime the cones are also null, but they certainly do not coincide. ${ }^{3}$

Now consider the second argument, that the choice of the present on the horizon is the only one satisfying a requirement of orthogonality to a local time direction. It is hard to make out what can be meant by "the" time direction since this of course differs per frame. One might suggest the temporal axis to be that of Schwarzschild coordinate time; after all this is one direction orthogonal to the horizon (null surfaces have the curious property that their normals are also tangent to them). But, being a light-like direction, no (massive) observer can identify the temporal direction of his restframe with this direction. A more plausible reading would be that, since the plane orthogonal to any local time direction lies in between the cones, it seems to be squashed onto the horizon (as suggested in Fig. 2) no matter what temporal direction we choose. But there is an easy way to see that this second reading cannot work. The light-like character of a surface is defined by its normal being everywhere light-like. The space-like character of a surface, in turn, is defined by its normal being everywhere time-like. Hence, there cannot be a time-like direction normal to a light-like

\footnotetext{
${ }^{3}$ If one needs conviction, simply consider that if the rays were to coincide, being light-like their inner product would be zero. But this inner product is a coordinate invariant, and it is easily seen not to be zero in a local inertial frame.
} 
horizon-the surface would have to be both light-like and space-like. And indeed, if we take proper heed of the non-degeneracy of the lightcones at the horizon as emphasised above, we can see that there is ample room for a spatial plane locally orthogonal to any temporal direction there - the situation is locally no different from the Minkowskian one.

Finally, let me digress briefly on this orthogonality requirement. I argued here only that local orthogonality to any single temporal direction can be had at the horizon. Of course, if we allow for crossing observers, no one hypersurface will be orthogonal to both of their worldlines at the event of crossing. And even if we do not allow for crossing observers, it is generally impossible to foliate a congruence of worldlines in an everywhere orthogonal way if rotation is present. (This is a special case of Froebenius' theorem; Malament (1995) explains this intuitively as the impossibility of slicing a rope in a way orthogonal to every fiber.) So even independent of considerations from black holes, the presentist might want to rethink the desideratum of worldline-orthogonality. Gödel found his infamous universe searching for solutions that exhibit this failure, where the rotation is that of the heavy bodies. Usually when the orthogonality condition is used to define a cosmic time, it is the heavy bodies the slicing is sought orthogonal to, not just any congruence of possible observer trajectories.

\subsection{How to slice a black hole}

If the above shows that there one can unproblematically find a present locally at the horizon, we may still wonder whether the same holds globally. Recall that we are investigating a traditional presentism here that demands a global spatial present. In fact, the Eddington-Finkelstein system mentioned above is everywhere regular and allows for a global spatial foliation. But the possibility for such a foliation is easier to see in yet another well-known coordinate system adopted to the Schwarzschild geometry: Kruskal-Szekeres coordinates. Let us show by construction that a global spatial slicing of a Schwarzschild black hole is indeed possible.

When we inspect the Schwarzschild metric in Kruskal-Szekeres coordinates we obtain the following:

$$
d s^{2}=\frac{32 M^{3}}{r} \mathrm{e}^{-r / 2 M}\left(-d v^{2}+d u^{2}\right)+r^{2} d \Omega .
$$

The Schwarzschild geometry is depicted in terms of these coordinates as in Fig. 4.

From Eq. 2.4 we immediately see that for radial directions $(d \Omega=0)$, setting $d v=$ $\pm d u$ yields $d s=0$. So a great benefit of these coordinates is that radial, light-like geodesics are always 45 degree lines in two-dimensional Kruskal-Szekeres diagrams (see Fig. 4). It can also be seen that we can foliate the whole of this geometry in a way such that every hyper-surface lies everywhere (including at the horizon) between the local future and past lightcones.

For instance, we may choose a $v=$ constant slicing. Inspecting the metric, we see that the induced metric on these hyper-surfaces $(d v=0)$ is everywhere positive. The slicing is therefore space-like. A presentist postulating this slicing will avoid 


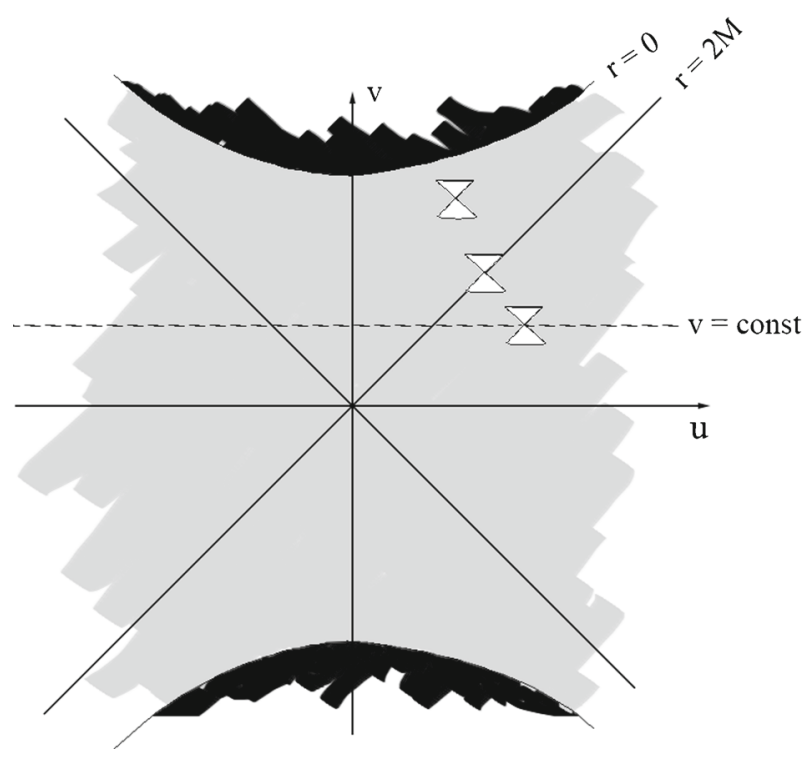

Fig. 4 The Schwarzschild geometry in Kruskal-Szekeres coordinates

the troubles that Romero and Peréz imagine. The choice is by no means unique: any slicing that is everywhere less than 45 degrees with respect to the $u$-axis in the diagram will also be everywhere space-like.

The conclusion for the Schwarzschild case is that a presentist here is indeed free to postulate a foliation in spacelike hypersurfaces such as his theory requires. In fact, there are many distinct foliations, equally suitable. This does lead to a certain embarrassment of riches: on what basis does the presentist choose one? Indeed there remains an air of arbitrariness, even ad hocness, attached to the postulation of a foliation where the physical theory requires none, more so because it requires breaking local relativistic symmetries by introducing a preferred spatial slicing. It could be argued then, that the presentist has work to do in the justification of the slicing of choice. ${ }^{4}$

\section{Trouble beyond the horizon?}

If the Schwarzschild metric is indeed, as argued above, compatible with a space-like foliation, does that mean that black holes pose no novel threat to presentism? That conclusion would be too quick. The Schwarzschild case is only the simplest of a

\footnotetext{
${ }^{4}$ Sometimes surfaces of constant mean curvature are considered (CMC) in the context of presentism, see Monton (2006) and Wüthrich (2010). I will not discuss these proposals because they are not specific to black holes, but note that CMC-foliations of Schwarzschild geometries seem available, see Brill et al. (1980). I thank an anonymous referee for pointing this out to me.
} 
range of geometries that can harbour some uncomfortable surprises. In the present section, I briefly sketch some of these surprises, bring out the issues they cause for presentism, and, leaning heavily on the authority of others, outline the way these issues are evaluated in current physics. Doing so will paint a more balanced view of the way the existence of black holes might impact presentist metaphysics.

\subsection{Rotating black holes: the Kerr geometry}

The Schwarzschild metric (2.1) can be seen as a limiting case of a more general geometry describing rotating black holes. The vacuum solution, known as the Kerr metric, was the result of a search for metrics that are not orthogonally foliable (Carter 1968), so we might expect a conflict with a desideratum of a present orthogonal to time. But the geometry turned out to contain a few more surprising features, which indeed pose a more direct threat to presentism. These features are relatively easily exhibited.

The Kerr metric in Boyer-Lindquist coordinates reads:

$$
\begin{aligned}
d s^{2}= & \left(-1+\frac{2 M r}{\rho^{2}}\right) d t^{2}+\frac{\rho^{2}}{\Delta} d r^{2}+\rho^{2} d \theta^{2} \\
& +\left(r^{2}+a^{2}+\frac{2 M r a^{2} \sin ^{2} \theta}{\rho^{2}}\right) \sin ^{2} \theta d \varphi^{2}-4 M r a \frac{\sin ^{2} \theta}{\rho^{2}} d t d \varphi .
\end{aligned}
$$

with

$$
\rho^{2}=r^{2}+a^{2} \cos ^{2} \theta
$$

and

$$
\Delta=r^{2}-2 M r+a^{2} .
$$

Here $a$ can be interpreted as the angular momentum of the black hole and $M$ as its mass. For $a=0$, this expression reduces to the Schwarzschild metric. The Kerr geometry is a strange beast. The exterior is used successfully in the modelling of astrophysical black holes, and is thought to describe their exterior final state uniquely. ${ }^{5}$ But the interior can become quite extravagant, as we will see below.

At first glance, the Boyer-Lindquist coordinates look somewhat like standard spherical coordinates plus a time direction on $\mathbb{R}^{4}$. But looks can be deceiving. First note that the metric expression diverges in two cases: when $\Delta=0$, and when $\rho=0$. If first we look at expression (3.3) for $\Delta$, we see that it equals zero at two values for $r{ }^{6}$ This turns out to give us not one, but two horizons. Like in the Schwarzschild

\footnotetext{
${ }^{5}$ This expectation derives from the famous "no-hair theorems". Note that the theorems are specific to the matter content considered, and there is no proof of a completely general uniqueness conjecture (Chrusciel et al. 2012). Note also that avoiding these theorems - say by modifications of general relativity, or by relaxing the stationarity assumption-may not solve the problems for the presentist discussed below, as closed time-like curves may still occur (Johannsen 2013).

${ }^{6}$ At least when $a<M$, or "slow Kerr", arguably the case of most physical interest (Carroll 2004). Fast Kerr, with $a>M$, is often considered unphysical because it contains naked singularities-famously conjectured away by Penrose. The problems for presentism would be similar to the ones discussed below for slow Kerr, only worse: there are no horizons and all of spacetime would be causally vicious.
} 
case, these degeneracies can be removed by transforming to other coordinates, and the metric can be extended to cover these horizons.

By contrast, the case $\rho=0$ gives us a true singularity. If we inspect expression (3.2) for $\rho$, we see that $r=0$ does not suffice to have $\rho=0$ : the metric only fails at $r=0$ when also $\theta=\pi / 2$. Suppose we were to stick to the interpretation of the Boyer-Lindquist coordinates as spherical coordinates on $\mathbb{R}^{4}$. Then, if geodesics were to approach $r=0$ from an angle other than $\theta=\pi / 2$, the curvature on them would not blow up; they would in fact be extendible. This shows the untenability of interpreting metric (3.1) as describing a geometry on $\mathbb{R}^{4}$ in standard spherical coordinates. In that interpretation, we would not have covered the whole manifold; indeed the manifold would be extendible. In fact $r=0$ does not describe a point but a disk, as $r=0$ does in ellipsoidal coordinates (one may write Minkowski spacetime in such coordinates; see Wald 1984). The metric fails only at the edge of the disk, for $r=0$ and $\theta=\pi / 2$. Since the metric is not singular at the inside of the disk, we may extend geodesics through it.

A further complication arises because the metric fails to be smooth across the coordinate disk enclosed by this ring. A smooth extension across this disk can be achieved by glueing two copies of $\mathbb{R}^{4}$ with the disk removed together, identifying the top of the removed disk of the one with the bottom of the removed disk of the other. Details of this extension can be found in Hawking and Ellis (1973). This way we can allow $r$ to take negative values, indeed range from $-\infty$ to $+\infty$. Whereas this would not make sense in the Minkowskian case, here we have a different manifold that can be meaningfully charted with a negative radius. We have found, then, a solution that lives on a manifold topologically quite different from, say, the Schwarzschild or Minkowskian case. One way to visualise this manifold is as in Fig. 5.

\subsection{Closed timelike curves}

But in these extended regions, an immediate problem for presentism is found. If we inspect the metric, we find that the longitudal direction $\partial_{\varphi}$ can become timelike in regions with small negative $r$. This can be seen relatively easily. The character of $\partial_{\varphi}$ is determined by $g_{\varphi \varphi}$ : it is timelike iff $g_{\varphi \varphi}<0$. The expression for $g_{\varphi \varphi}$,

$$
g_{\varphi \varphi}=\left(r^{2}+a^{2}+\frac{2 M r a^{2} \sin ^{2} \theta}{\rho^{2}}\right) \sin ^{2} \theta,
$$

reduces on the equatorial plane $\left(\theta=\frac{1}{2} \pi\right)$ to

$$
g_{\varphi \varphi}=r^{2}+a^{2}+\frac{2 M a^{2}}{r} .
$$

This is negative for small negative $r$. The direction, then, is time-like there. But integral curves for the direction $\partial_{\varphi}$ are closed curves circling the axis of rotation. For small negative $r$, then, these are closed time-like curves. The situation is often visualised as the lightcones tilting over in direction of rotation, eventually sufficiently so 


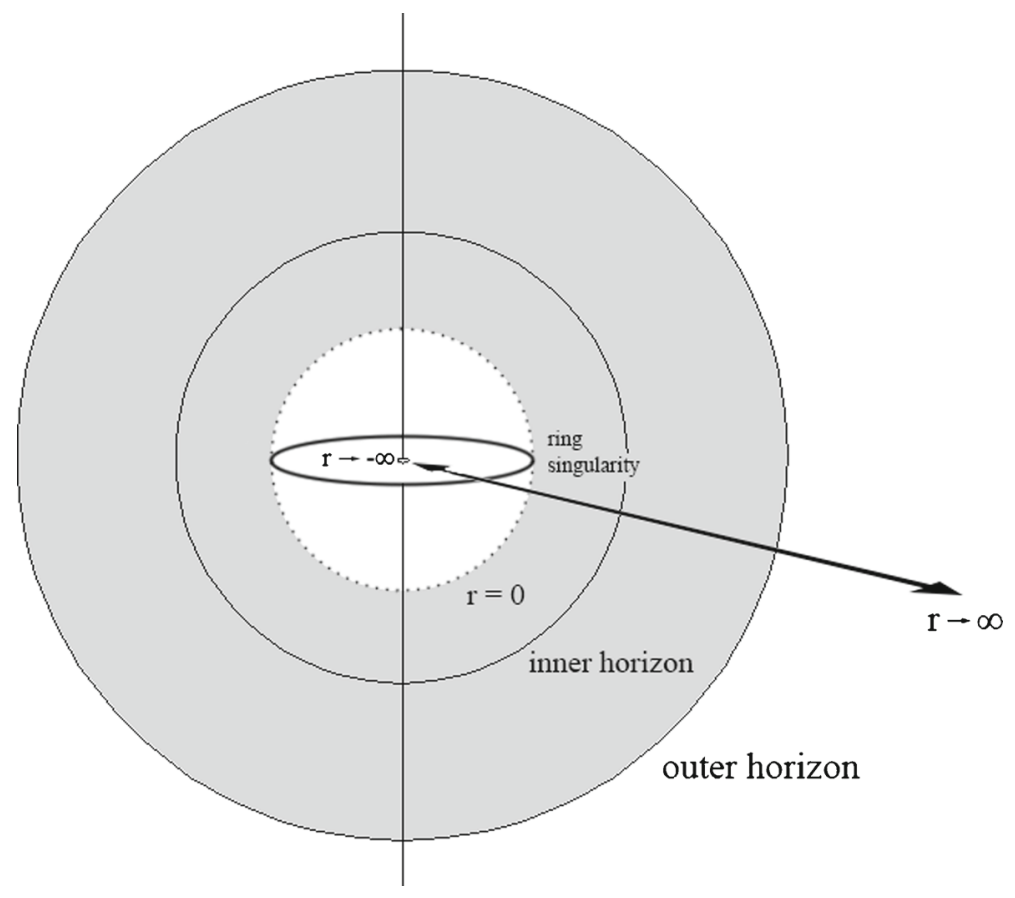

Fig. 5 Visualisation of the Kerr interior; adapted from O'Neill (2014). The $r=0$ disk here is the surface of a sphere, with each side of the disk a hemisphere. The extension through the disk is included as a negative- $r$ sphere, with $r$ drawn exponentially. So, $r=-\infty$ lies at the centre, and the region is as large as the positive $r$ region

to include this direction in their futures (but see Andréka et al. (2008) for qualification of this picture). Following the direction $\partial_{\varphi}$ around, a curve can enter into its past once again.

Note that these curves are not "unrollable", that is, they cannot be removed by going to a larger covering space. By contrast, we could obtain closed time-like curves by "rolling up" Minkowski spacetime along a space-like direction. It may be argued that presentism could live with such closed time-like curves-we would obtain a cyclic time that could still parametrise a space-like foliation hospitable to presentism. Any such curve intersects the space-like hypersurfaces only once, and, as Monton (2003) argues, this could be interpreted as an eternal-recurrence scenario not necessarily in conflict with presentism. Technically, the saving grace is that these more benign curves are not contractible to a point (not homotopic to zero). But those in the Kerr case are. Carter (1968) considers the options one would have for preventing this contractibility. One might want to remove the ring-singularity from the space to obtain a space that is not simply connected, and allows curves around the ringsingularity that are not contractible. But the closed time-like curves in our geometry do not wind around the ring-singularity, so this would be of no help. To prevent the 
example curve from being contractible, one would have to remove (for instance) the axis-but there is no physical reason to do so. ${ }^{7}$

Perhaps it is intuitively obvious that such closed time-like curves are hard to swallow for the presentist, but one can make the trouble explicit in the following way. Start from the observation (Carter 1968) that every contractible closed time-like curve must cross a global space-like surface an even number of times. We can parametrise the presentist's foliation by such surfaces with a global time coordinate $T$. We can also parametrise the closed time-like curve with a parameter $\lambda$. Now if at one crossing point, the curve will cross in positive time direction $(d T / d \lambda>0)$, there will be another where it will cross in negative global time direction $(d T / d \lambda<0)$.

If this-processes along the curve will evolve in reverse global time-does not spell enough trouble, consider this. If the curve is sufficiently smooth (as our earlier circular example curve is), then somewhere between the crossing points, $d T / d \lambda$ will have to go through zero to change sign. That means that at some point, global time $T$ will not increase along the curve. But that means that its tangent there is space-like: it means that the tangent there lies in a surface assumed to be space-like. But by assumption the tangent was time-like (it is a time-like curve). So we have a contradiction.

When we look back at the visualisation in Fig. 5, we now see that it cannot be understood as representing a spatial slice at a moment in time: some of the distances in this figure are time-like. And since there will be such time-like distances in at least some slices of any foliation of the Kerr geometry, this geometry cannot accommodate a presentist metaphysics that postulates an everywhere space-like foliation.

\subsection{Threat evaluation for presentists}

How problematic for presentism the presence of closed time-like curves in a solution is, depends of course on how seriously we should take that solution. The causality violating region in Kerr spacetime lies in an extension through the singularity to negative $r$ values. ${ }^{8}$ This is a region as large as the positive $r$ region, indeed a parallel universe, with peculiar features such as a reversal of the metric's gravitational field (the region inside the inner horizon is sometimes referred to as a "white hole" region). And indeed it is hard to imagine moving from a manifold with Minkowski-like $\mathscr{J}^{-}$ (distant past) in which a star begins its collapse, to a topologically different one-one might have to postulate a pre-established harmony of coalescing parallel universes ${ }^{9}$ (for speculation on a different, wormhole-like scenario, see Charlton and Clarke 1990). The maximal extension of Kerr spacetime becomes stranger still. The horizons divide the spacetime in so-called Boyer-Lindquist blocks, and by a copy-and-paste process we can assemble these into an endless patchwork of - traversable-parallel

\footnotetext{
${ }^{7}$ Furthermore, Carter argues that removing the axis would not even cure the spacetime of all closed timelike curves: we could still obtain small such curves that briefly dip in and out of the time-machine region"thus making up for lost time"-without circling around the axis.

${ }^{8}$ However, for charged black holes, described by the family of Kerr-Newman metrics, Carter (1968) mentions that the problematic region may spill over into positive $r$.

${ }^{9}$ See Misner et al. (1973) for visualisations of the analogous case in the maximal Schwarzschild extension.
} 
universes that together form the maximally extended Kerr spacetime (see O'Neill 2014 for exposition).

It is not surprising then, that many authoritative voices echo the sentiment that somewhere in these regions, the Kerr metric must depart from actuality (see Misner et al. (1973) p883, Carroll (2004) p266, O’Neill (2014) p103, Wald (1984) p317). Perhaps the presentist may find strength in such allies, operating on a methodological principle that metaphysics need only conform to actual physics. Discussion of such principles are familiar from the context of Gödelian universes (Dorato 2002), so I will here focus on the physical arguments against actuality in this case. Some of the reasons for not taking the problematic region as a realistic description of the actual universe are the following. For starters, wherever matter fields are present, the solution will depart from the given vacuum solution. The Schwarzschild case is also maximally extendible into a spacetime containing a white hole and a nontraversable wormhole connecting to a parallel universe. These regions, however, are usually not considered realistic because they will be replaced by the matter of the collapsing star as it turns into a black hole. The Kerr case is more difficult. It is not spherically symmetrical, and only in the asymptotic future will the geometry settle down to a stationary state. Whereas there are arguments to the effect that the exterior solution will eventually resemble Kerr, ${ }^{10}$ these do not hold for the interior. Wald compares the case tentatively to the spherically symmetric case of a charged nonrotating black hole (Reissner-Nordström geometry). In that case, there remains a nonnegligible possibility that for certain choices of parameters, the collapsing matter will leave some of the problematic regions uncovered. But there are reasons to suspect that physical fields will diverge at the inner horizon which will produce singular behaviour there preventing such further evolution. Similar phenomena are thought to occur in a realistic Kerr-like collapse. ${ }^{11}$

There are more general reasons to object to the development of closed time-like curves than merely a taste for presentism. Because such acausalities bring with them a host of problems not just for the traditional metaphysician, but also for the working physicist, their elimination has also long been on that physicist's wish-list. These issues range from the existence and nature of consistency constraints on the fields living on such curves (see Earman 1995) to energy conditions thought to be violated in their development (see Smeenk and Wüthrich 2011). There is an important connection to ongoing research on singularities in general relativity (and beyond, see Earman and Wüthrich 2013). If closed time-like curves do develop in a region of

\footnotetext{
${ }^{10}$ See footnote 5 .

${ }^{11}$ Sometimes the existence of a Cauchy horizon, necessarily associated with the development of acausalities, is cited as a ground for not taking the causality violating region seriously within general relativity (Visser 2003). While it is off course a nontrivial issue how to even formulate the dependence of the acausal region beyond a Cauchy horizon on the causal region before it, the problem does not seem to be insurmountable, and it is too quick to write off the existence of the extensions on these grounds (See Smeenk and Wüthrich 2011 for discussion). Penrose, however, in 1968 went further and first argued for an infinite blueshift at the inner horizon preventing the development of the acausal region. See Israel (2003) for an informal account; the exact nature of the behaviour at the Cauchy horizon is still under debate (see for example Dafermos 2008).
} 
spacetime that is otherwise causal, the spacetime will have a Cauchy horizon: a horizon beyond which prediction from initial data as specified on an earlier hyper-surface fails. Since such failure is also a feature of spacetimes with singularities, certain arguments against singularities (strong cosmic censorship, as opposed to weak censorship of naked singularities, which would only hide the pathologies behind a horizon) would also work against closed time-like curves (see Smeenk and Wüthrich 2011). Other theorems purport to show that any spacetime has a maximal extension without closed time-like curves in the future, thus blocking the necessity of their development (the theorem is Krasnikov's, and is contested by Manchak, as discussed in Smeenk and Wüthrich 2011). Most directly, Hawking's 1992 chronology protection conjecture argues precisely against the existence of Cauchy horizons, and hence closed timelike curves. ${ }^{12}$ If successfully developed into a theorem, this conjecture would make the universe a safer place not only for historians, but also for presentists. Thus far, none of these attempts are thought to be entirely successful (see Earman et al. 2009). But the presentist might draw courage from the fact that his fight is part of a larger battle where he may find forces to join against a common enemy.

\section{Conclusion}

Reconciliations between presentism and modern spacetime physics, even if possible in the letter, often smack contrary to the relativistic spirit. It seems unlikely that relativity and presentism will ever enter into a happy marriage. ${ }^{13}$ However, we have seen above that the case for a definitive split on the basis of the encounter with black holes is inconclusive. First we have seen that, contrary to arguments by Romero and Pérez, Schwarzschild black holes pose no new independent threat to presentism. The case of rotating black holes proved more intricate. There may be insurmountable trouble lurking in their interior, but we have seen that it is as yet hard to evaluate its eventual metaphysical impact, precisely because the physicist's verdict is still out. Strange things happen in black holes, and much of it is inextricably connected to the future developments of physics. ${ }^{14}$ We have seen that the fate of presentism is likewise intimately connected to these developments. It is, then, too soon to write off presentism as a viable metaphysical candidate, but a close scrutiny of developments on the frontiers of physics will be needed to see whether it will remain a live possibility.

Acknowledgments Let me thank all in the project 'The Structure of Reality and the Reality of Structure', Gijs Leegwater, Stefan Wintein and F.A. Muller, for helpful discussion, and the Netherlands Organisation for Scientific Research (NWO) for funding that project. I also thank two anonymous referees for useful comments.

\footnotetext{
${ }^{12}$ See Visser (2003) for an informal review of arguments leading to the conviction that a full understanding of chronology horizons will require a quantum theory of gravity; see also Earman and Wüthrich (2013).

${ }^{13}$ There have been arguments for Monton (2006) and against Wüthrich (2010) the hope of finding a more hospitable environment for presentism in theories of quantum gravity. While it is true that there are programs employing a foliation (see for instance Hořava 2009), it is hard to place any reliable bet in this field.

${ }^{14}$ Of course the black hole physics I have discussed here is relatively old-hat: speculation of firewalls and fuzzballs closer to the frontiers may, when more firmly established, require us to rethink my evaluation in unforeseeable ways.
} 
Open Access This article is distributed under the terms of the Creative Commons Attribution 4.0 International License (http://creativecommons.org/licenses/by/4.0/), which permits unrestricted use, distribution, and reproduction in any medium, provided you give appropriate credit to the original author(s) and the source, provide a link to the Creative Commons license, and indicate if changes were made.

\section{References}

Andréka, H., Németi, I., \& Wüthrich, C. (2008). A twist in the geometry of rotating black holes: seeking the cause of acausality. general Relativity and Gravitation, 40(9), 1809-1823.

Bourne, C. (2006). A future for presentism. Oxford: Clarendon Press.

Brill, D.R., Cavallo, J.M., \& Isenberg, J.A. (1980). K-surfaces in the Schwarzschild space-time and the construction of lattice cosmologies. Journal of Mathematical Physics, 21(12).

Carroll, S.M. (2004). Spacetime and geometry. San Francisco: Addison Wesley.

Carter, B. (1968). Global structure of the Kerr family of gravitational fields. Physical Review, 174(5), 1559-1571.

Charlton, N., \& Clarke, C.J.S. (1990). On the outcome of Kerr-like collapse. Classical and Quantum Gravity, 743-749.

Chrusciel, P.T., Costa, J.L., \& Heusler, M. (2012). Stationary black holes: uniqueness and beyond. Living Reviews Relativity, 15(7).

Crisp, T. (2003). Presentism. In Loux, M.J., \& Zimmerman, D.W. (Eds.), The Oxford handbook of metaphysics. Oxford: Oxford University Press.

Dafermos, M. (2008). Price's law, mass inflation and strong cosmic censorship. arXiv:gr-qc/0401121v1.

Dorato, M. (2002). On becoming, cosmic time and rotating universes. In Callender, C. (Ed.), Time, Reality and Experience. Cambridge: Cambridge University Press.

Earman, J. (1995). Bangs, crunches, whimpers and shrieks. Oxford: Oxford University Press.

Earman, J., Smeenk, C., \& Wüthrich, C. (2009). Do the laws of physics forbid the operation of time machines? Synthese, 169, 91-124.

Earman, J., \& Wüthrich, C. (2013). Time machines. In Zalta, E.N. (Ed.), The Stanford Encyclopedia of Philosophy. Winter 2013 edition.

Gödel, K. (1949). A remark about the relationship between relativity theory and idealistic philosophy. In Schilpp, P.A. (Ed.), Albert Einstein: philosopher-scientist. New York: Harper and Row.

Hawking, S.W., \& Ellis, G.F.R. (1973). The large scale structure of space-time. Cambridge: Cambridge University Press.

Hořava, P. (2009). Quantum gravity at a Lifshitz point. Physical Review D, 79.

Israel, W. (2003). Black hole uniqueness and the inner horizon stability problem. In Gibbons, G.W., Shellard, E.P.S., \& Rankin, S.J. (Eds.), The fututre of theoretical physics and cosmology. Cambridge: Cambridge University Press.

Johannsen, T. (2013). Systematic study of event horizons and pathologies of parametrically deformed Kerr spacetimes. arXiv:gr-qc/1304.7786v3.

Malament, D. (1995). Intorductory note to 1949b. In Feferman, S.e.a. (Ed.), Kurt Gödel, collected works vol. III. Oxford: Oxford University Press.

Misner, C.W., Thorne, K.S., \& Wheeler, J.A. (1973). Gravitation. New York: W.H. Freeman and company.

Monton, B. (2003). Presentists can believe in closed timelike curves. Analysis, 63, 199-203.

Monton, B. (2006). Presentism and quantum gravity. In Dieks, D. (Ed.), The ontology of spacetime. Amsterdam: Elsevier.

O'Neill, B. (2014). The geometry of Kerr black holes. Mineola, New York: Dover Publications.

Rakic, N. (1997). Past, present, future, and special relativity. British Journal for the Philosophy of Science, 48, 257-280.

Romero, G.E., \& Pérez, D. (2014). Presentism meets black holes. European Journal for Philosophy of Science, 4, 293-308.

Smeenk, C., \& Wüthrich, C. (2011). Time travel and time machines. In Callender, C. (Ed.), The Oxford handbook of philosophy of time. Oxford: Oxford University Press.

Visser, M. (2003). The quantum physics of chronology protection. In Gibbons, G.W., Shellard, E.P.S., \& Rankin, S.J. (Eds.), The Fututre of Theoretical Physics and Cosmology. Cambridge: Cambridge University Press.

Wald, R.M. (1984). General relativity. Chicago: University of Chicago Press.

Wüthrich, C. (2010). No presentism in quantum gravity. In Petkov, V. (Ed.), Space, time and spacetime. Berlin: Springer. 